\title{
Quantifying the clinical characteristics of coronavirus disease 2019 (COVID-19) of different age segments based on 60 patients
}

\section{Gao Weiwei}

Nanjing Second Hospital

\section{Zhang Xia}

Nanjing Second Hospital

Hu Zhiliang

Nanjing Second Hospital

Chi Yun

Nanjing Second Hospital

Zheng Yi shan

Nanjing Second Hospital

Hu Chun mei

Nanjing Second Hospital

Yi Yong Xiang

Nanjing Second Hospital

Zeng Yi ( $\nabla$ njyy002@njucm.edu.cn )

Nanjing Second Hospital

\section{Research article}

Keywords: coronavirus disease 2019, COVID-19, clinical characteristics, lung,age

Posted Date: July 17th, 2020

DOI: https://doi.org/10.21203/rs.3.rs-20112/v2

License: (c) (i) This work is licensed under a Creative Commons Attribution 4.0 International License. Read Full License 


\section{Abstract}

Background: Novel coronavirus disease 2019 (COVID-19) has spread around the world; therefore, more attention should be paid to the clinical features of COVID-19, with the aim of improving the diagnosis and treatment of patients. Methods: By 15 February 2020, 60 patients diagnosed with COVID-19 had been admitted to Nanjing Second Hospital. We analyzed the clinical features of different age segments infected with COVID-19 prospectively, including epidemiological, clinical, laboratory, and radiological characteristics; treatment, clinical outcomes, and prognosis of this cohort of patients.

Results: The cohort comprised 29 male and 31 female patients (median age $=46.18$ years old (range: 18-97). Fifty-five (91.7\%) patients had a clear epidemiological contact history. The average incubation period was 7.92 days. The most common clinical manifestations were fever $(85 \%)$ and cough $(75 \%)$. Peripheral white blood cell counts were mostly normal at admission, 7 days, and 14 days, with no differences among patients of different ages. The lymphocyte counts of all patients were in the normal range on admission, and after 7 days and 14 days of treatment; however, the lymphocyte count in $>65-$ year-old patients was less than that in the $<40$ and 40-65-year-old groups after 7 and 14 days of treatment $(P<0.05$, respectively). At admission, the CD4 T lymphocyte count was within the normal range; however, the CD4 T lymphocyte count in $>65$-year-old group was less than that in the $<40$ and $40-$ 65 -years-old groups after 14 days of treatment. The CD4 T lymphocyte counts were $723.46 \pm 243.82 / \mathrm{ml}$ $(<40), 640.00 \pm 242.30 / \mathrm{ml}(40-65)$, and $399.88 \pm 256.16 / \mathrm{ml}(>65)(P=0.0075)$. The $>65$-years-old group had higher levels of lactate dehydrogenase $(269.83 \pm 73.36 \mathrm{vs}$. $208.52 \pm 35.67$ and $243.83 \pm 76.66)$ after 14 days $(P=0.0496)$. Imaging revealed more lesions in the $40-65$ and $>65$-year-old groups $(P<0.0001)$. The days after the nucleic acid detection turned negative in the three age groups were: $9.19 \pm 3.93(<40)$, $10.04 \pm 4.10(40-65)$, and $13.57 \pm 2.76(>65)(P=0.0373)$. After antiviral treatment, together with antiinfection regimen if the patient with lung infection and continuous oxygen inhalation if the patient is hypoxic, all patients achieved total recovery and were discharged with follow-up.

Conclusion: Patients with COVID-19 pneumonia generally had an epidemiological history. Older patients showed more extensive lesions upon admission, more severe illness, slower recovery of immune function, the longer viral nucleic acid persistence.

\section{Background}

Recently, a novel coronavirus has emerged from Wuhan, a city in central China that has an estimated population of about 10 million and is a major transportation hub. Within a few weeks, the novel coronavirus, tentatively named as Severe acute respiratory syndrome coronavirus 2 (SARS-CoV-2) that causes coronavirus disease 2019 (COVID-19), was announced by the World Health Organization [1]. Coronavirus is a large family of RNA viruses that can be transmitted through droplets or contact, and might also be transmitted through the fecal-oral route, with a high incidence and rapid infection [2]. SARSCoV-2 could be considered as a distinct coronavirus from SARS. The genome of SARS-CoV- 2 has $89 \%$ nucleotide identity with bat SARS-like-CoVZXC21 and $82 \%$ with that of human SARS-CoV $[3,4]$, 
suggesting that it was probably transmitted from bats or another host after developing the ability to infect humans [5]. As the virus continues to spread, COVID-19 threatens not only China, but also the rest of the world due to its international spread via travelers, posing a huge threat to global public health. Nanjing is also a high-incidence city, and the Nanjing Public Health Medical Center (Jiangsu Provincial Infectious Diseases Hospital) is a designated site for provincial infectious diseases, which has shouldered the heavy responsibility of treating such patients. Up to 15 February 2020, 60 patients diagnosed with COVID-19 had been admitted to Nanjing Second Hospital. In the present study, we prospectively collected and analyzed the epidemiological, clinical, laboratory, and radiological characteristics; and the treatment and clinical outcomes of these patients. We also stratified these data according three patient age groups ( $<40$ years old, 40-65 years old, and $>65$ years old) to increase awareness of the clinical features of COVID-19, and to improve the diagnosis and treatment of patients.

\section{Methods}

\section{Patients and parameters}

Up to 15 February 2020, 60 patients diagnosed with COVID-19 infection were collected. We prospectively collected and analyzed the epidemiological, clinical, laboratory, and radiological characteristics; and the treatment and clinical outcomes of three patient age groups ( $<40$ years old, 40-65 years old, and $>65$ years old), and summarized their clinical characteristics and prognosis.. For imaging evaluation, we used high resolution computed tomography (HRCT) scans, performed on the day of admission, and rechecked every 7 days after treatment. The patients were treated according to the recommendations of the Diagnosis and Treatment Plan for Novel Coronavirus-infected Pneumonia (Fifth Trial Edition) issued by the National Health Commission. The treatment included lopinavir/ritonavir (500 mg, po, twice/day) for antiviral treatment, together with an anti-infection regimen if the patient had a lung infection, and continuous nasal cannula oxygen inhalation at $3 \mathrm{~L} / \mathrm{min}$ if the patient was hypoxic). Ethics approval and written consent to participate was exempted by the institutional review boards of Nanjing Second Hospital because all case-based data utilized in the study were collected routinely. All data were fully anonymized before analysis. These patients have not been reported previously in any other submission by our team or anyone else.

Statistical Analysis

Classification variables are expressed as frequencies or percentages. For normally distributed data, measurements are expressed as the mean $\pm S D$, and significance was detected using the chi squared test or Fisher's exact test. The quantified variables of the parameters are expressed as the mean $\pm S D$, and the significance was tested using a t-test. $P<0.05$ was considered statistically significant in all statistical analyses. SPSS statistical software (Macintosh version 26.0, IBM, Armonk, NY, USA) was used for the statistical analysis.

\section{Results}


The 60 patients were diagnosed with COVID-19 using real-time reverse transcription (RT)-PCR and were admitted to Nanjing Second Hospital. All patients satisfied the probable fifth edition COVID-19 criteria recommended by the World Health Organization. The study included 29 male and 31 female patients, whose median age was 46.18 (range: 18-97) years old. Forty (66.67\%) of these patients had no underlying diseases. The other 20 patients had underlying diseases: Eight suffered from diabetes, six suffered from chronic obstructive pulmonary disease (COPD), and six suffered from hypertension. Fiftyfive $(91.7 \%)$ patients had a clear epidemiological contact history, including a recent trip to Wuhan and close contact with patients with pneumonia, which included 36 patients in 6 family clusters. The average incubation period was 7.92 days. The most common clinical manifestations were fever (85\%) and cough (75\%), followed by chest tightness (36.7\%) and sore throat (33.3\%). The laboratory test results showed that the patients' leukocyte counts were mostly normal at admission, 7 days, and 14 days, with no difference among patients of different ages $(P>$

0.05 , respectively). Among the whole patient cohort, the lymphocyte count was within the normal range on admission, and after 7 days and 14 days of treatment; however, after grouping by age, we found that the lymphocyte count in the $>65$-year-old patients was less than that in the $<40$-year-old and $40-65$-yearold patients after 7 and 14 days of treatment $(0.78 \pm 0.28,0.97 \pm 0.37$ (range: $0.80-4.00$ ), both $P<0.05$, respectively). The total CD4 T lymphocyte count was in the normal range on admission and after 14 days of treatment ( $537.34 \pm 312.16,644.29 \pm 262.82$, range $414-1123 / \mathrm{ml}$, respectively); however, in the older patients ( $>65$ years old), the CD4 T lymphocyte count was significantly lower than that in the young and middle-aged patients $(723.46 \pm 243.82,640.00 \pm 242.30,399.88 \pm 256.16 / \mathrm{ml})$ in the < 40-year-old, 40-65year-old, and $>65$-year-old patients, respectively, both $P=0.0075$ ). Patients aged $>65$ years old had higher levels of lactate dehydrogenase (LDH) $(269.83 \pm 73.36$ vs. $208.52 \pm 35.67$ and $243.83 \pm$ $76.66(\mathrm{IU} / \mathrm{L})$ in the other two groups, $P=0.0496)$ after 14 days of treatment. Arterial blood gas analysis showed lower scores among the 40-65-year-old and the $>65$-year-old patients than in the $<40$-year-old patients $(P<0.05$, respectively). The imaging distribution on admission showed more lesions in the $40-$ 65 -year-old and $>65$-year-old patients compared with those in the $<40$-year-old patients $(P<0.0001)$. The overall number of days until nucleic acid detection turned negative was 10.11 days: $9.19 \pm 3.93(<40$ years old), $10.04 \pm 4.10$ ( $40-65$ years old), and $13.57 \pm 2.76$ ( $>65$ years old) $(P=0.0373)$. After treatment, comprising antivirals plus an anti-infection regimen if the patient had a lung infection and antivirals plus continuous oxygen inhalation if the patient was hypoxic, our patients achieved total recovery and were discharged with follow-up and home isolation without any serious complications, such as vital organ dysfunction or the need for mechanical ventilation. The overall clinical manifestation and laboratory results of the patients are described in Table 1.

\section{Discussion}

In the present study, we reported the analysis of a cohort of 60 patients with laboratory-confirmed COVID19. There were no differences between the male and female patients. Fever was the most common symptom ( $85 \%)$, followed by cough without sputum, chest tightness, and sore throat, which were consistent with current reports concerning COVID-19 pneumonia [6-8]. These clinical features are similar 
to other respiratory infections, making it difficult to identify COVID-19, because most patients presented with upper respiratory tract signs and symptoms, such as fever, dry cough, and dyspnea. Most patients (55 (91.7\%)) had a clear epidemiological contact history, including recent travel to Wuhan or close contact patients with COVID-19 infection. Among these patients, which included 36 patients in 6 family clusters, most manifested as one confirmed patient in the family, with others being diagnosed subsequently, including some asymptomatic patients. The results demonstrated the obvious infectivity of this disease and the importance of isolation [9]. A previously study [10] found that patients with underlying diseases are more likely to develop severe COVID-19. A recent study [11] showed that the occurrence of COPD was associated with a nearly four-fold higher risk of developing severe COVID-19. In the present study, we found that most patients (66.67\%) had no underlying disease, and only six patients (10\%) suffered from chronic obstructive pulmonary disease (COPD) related to the lungs (the virus tends to attack peripheral vasculature and bronchus in the early stage of the disease). Notably, there was no significant correlation between suffering from COVID-19 and the patient's underlying disease; however, the infection might progress faster in patients with underlying diseases than in those without. Older patients had a longer incubation period and tended to develop more severe disease, indicating that the older the patient, the worse their condition becomes. Analysis of the leukocyte count indicated that white blood cells have no obvious involvement in, or correlation with, the immune process of the disease; however, it is also possible that the patients in this group are all imported patients with relatively mild illness [12]. Earlier studies have shown that lymphopenia is a typical laboratory abnormality observed during highly pathogenic coronavirus infections $[13,14]$, such as severe acute respiratory syndrome coronavirus (SARS-CoV) and Middle East respiratory syndrome coronavirus (MERS-CoV) infections $[15,16]$. We observed that the lymphocyte count in the $>65$-year-old patients was less than that in the < 40-year-old and 40-65-years-old patients after 7 and 14 days of treatments. In the early stage of the disease, the immune function is not activated, but the immune system begins to activate after treatment, resulting in the decline of lymphocytes, which might be ascribed to their weak immune system. Our findings support those of previous reports suggesting that older age and lymphopenia are potential risk factors for severe COVID-19. Further studies are warranted to determine the mechanism of lymphopenia's involvement in the pathogenesis of COVID-19, which might lead to treatments to prevent COVID-19 exacerbation. Other reports showed that the extent of CD4 T lymphopenia was higher in patients with severe COVID-19 disease than in those with in mild disease, and is associated with disease severity $[17,18]$. The CD4 T lymphocyte count was significantly lower in the elderly patients than in the middleaged and young patients, which might be related to the virus attacking the target organ at the early stage, whereas in the later period, it starts to attack the body's immune system and causes T lymphocyte apoptosis or bone marrow suppression, which is more obvious in the middle-aged and elderly patients $[19,20]$. Chest high-resolution computed tomography (HRCT) is an important screening tool for COVID-19 because of its high sensitivity and convenience. The imaging manifestations of COVID-19 pneumonia are similar to those of common viral pneumonia, but also have their own imaging characteristics. The most common manifestations of COVID-19 pneumonia in the early stage are patchy ground glass opacities, mainly distributed in the sub-pleural area [21-23]. At disease onset, the number of affected lobes on admission in elderly patients was higher (Fig. 1A,B), and the disease progressed faster than it did in 
young patients after 7 days of treatment (Fig. 1C,D). In the present study, the lesions mainly involved 1-2 lung fields and 2-4 lung fields, with a higher number of lesions in the lungs of older patients.

The sequence of the SARS-CoV-2 receptor binding domain (RBD), including its receptor-binding motif (RBM) that directly contacts angiotensin I converting enzyme 2 (ACE2), is similar to that of SARS-CoV, strongly suggesting that SARS-CoV-2 uses ACE2 as its receptor [24].These findings provide the basis for starting further studies on the pathogenesis of the virus, and studies to optimize the design of diagnostic, antiviral, and vaccination strategies for this emerging infection. Recently, the COVID-19 has been employed as an anti-HIV treatment and has been combined with interferon to treat. Most patients with COVID-19 in our hospital received Abidol or Lopinavir antiviral therapy, or intravenous methylprednisolone in some severe patients. In the present study, the average number of days after the nucleic acid test turned negative after treatment was 10.11, with the oldest patients taking the longest to test negative for viral nucleic acids. The number days of for nucleic acid turn negative in older patients was significantly longer than that in the middle-aged and young patients $(13.57 \pm 2.76$ vs. $10.04 \pm 4.10$ and $9.19 \pm 3.93$, respectively). Indicating that the older the patient, the longer the treatment cycle.

The purpose of this study was to observe the clinical characteristics of the novel coronavirus pneumonia and to participate in controlling the outbreak by effective countermeasures. This analysis has limitations. First, this clinical research involved a relatively small number of cases. Second, the data analyzed in this study were from the early phase of the outbreak and we lack follow-up data concerning long-term prognosis and complications. Our team will continue to follow up these patients in terms of their prognosis and long-term complications.

\section{Conclusion}

The patents with coronavirus disease 2019 pneumonia in this study generally had an epidemiological history. The most common clinical manifestations are fever and cough without sputum. Older patients had more extensive the lesions upon admission, a higher proportion of severe illness, slower recovery of immune function, and a longer number of days nucleic acid turn egative after treatment .

\section{Abbreviations}

COVID-19: coronavirus disease 2019; COPD: chronic obstructive pulmonary disease; LDH: lactate dehydrogenase; SARS-CoV: severe acute respiratory syndrome coronavirus; MERS-CoV: Middle East respiratory syndrome coronavirus; HRCT: high-resolution computed tomography ;RBD: receptor binding domain; RBM: receptor-binding motif; ACE2: angiotensin I converting enzyme 2

\section{Declarations}

\section{Acknowledgements}

Not applicable. 


\section{Authors' contributions}

WWG conducted the literature review and wrote the draft. YZ and YXY conceived the study and revised the manuscript. All authors have read and approved the manuscript.

\section{Funding}

The authors received no specific funding for this work.

\section{Ethics approval and consent to participate}

Ethics approval and consent to participate written consent was exempted by the institutional review boards of the Second Hospital of Nanjing as all case-based data utilized in the study are routinely collected. All data were fully anonymized before analysis.

\section{Consent to publication}

Not applicable.

\section{Competing interests}

The authors declare that they have no competing interests.

\section{Availability of data and materials}

Data relating to this study are contained and presented in this document. Other materials are available from the corresponding authors on reasonable request.

\section{Authors' Information}

Wei-Wei Gao, Department of Tuberculosis, Affiliated Hospital of Nanjing University of Chinese Medicine, The Second Hospital of Nanjing, Nanjing Municipal Public Health Medical Center, Nanjing, Jiangsu 210003, P.R. China, Physician, Email: weiweigao1106@163.com, Mobile Number: 15251856076.

Yi Zeng, Department of Tuberculosis, Affiliated Hospital of Nanjing University of Chinese Medicine, The Second Hospital of Nanjing, Nanjing Municipal Public Health Medical Center, Nanjing, Jiangsu 210003, P.R. China, Master, Chief Physician, Email: njyy002@njucm.edu.cn.Mobile Number: 13601586598.

Zhiliang Hu,Department of infectious Disease,Affiliated Hospital of Nanjing University of Chinese Medicine, The Second Hospital of Nanjing, Nanjing Municipal Public Health Medical Center, Nanjing, Jiangsu 210003, P.R. China,Doctor,AssociateChief Physician, Email:huzhiliangseu@163.com.

Yun Chi,Department of infectious Disease ,Affiliated Hospital of Nanjing University of Chinese Medicine, The Second Hospital of Nanjing, Nanjing Municipal Public Health Medical Center, Nanjing, Jiangsu 210003, P.R. China,Master冈Chief Physician,Email:shuiyeyun@126.com. 
Yishan Zheng,Department of Intensive Medicine,Affiliated Hospital of Nanjing University of Chinese Medicine, The Second Hospital of Nanjing, Nanjing Municipal Public Health Medical Center, Nanjing, Jiangsu 210003, P.R. China,Doctor,Chief Physician,Email:zhengyishan@njucm.edu.cn.

Chunmei Hu,Department of Tuberculosis, Affiliated Hospital of Nanjing University of Chinese Medicine, The Second Hospital of Nanjing, Nanjing Municipal Public Health Medical Center, Nanjing, Jiangsu 210003, P.R. China,Doctor,AssociateChief Physician,Email:zhengyishan@njucm.edu.cn.

Yongxiang Yi,Affiliated Hospital of Nanjing University of Chinese Medicine, The Second Hospital of Nanjing, Nanjing Municipal Public Health Medical Center, Nanjing, Jiangsu 210003, P.R. China,Doctor,AssociateChief Physician,ian0126@126.com.

Xia Zhang,University of Chinese Medicine, The Second Hospital of Nanjing, Nanjing Municipal Public Health Medical Center, Nanjing, Jiangsu 210003, P.R. China,Chief

Physician,Email:zhangxia365@sina.com.

\section{References}

1. World Health Organization. Situation report-23, Novel Coronavirus COVID-19, 12 February, 2020. https://www.who.int/emergencies/diseases/novel-coronavirus-2019/situation-reports.

2. Guan WJ, Ni ZY, Hu Y, Liang WH, Ou CQ, He JX, et al. Clinical characteristics of coronavirus disease 2019 in China. N Engl J Med. 2020;382(18):1708-20.

3. Chan JF, Kok KH, Zhu Z, Chu H, To KK, Yuan S, et al. Genomic characterization of the 2019 novel human-pathogenic coronavirus isolated from a patient with atypical pneumonia after visiting Wuhan. Emerg Microbes Infect.2020;9(1):221-36.

4. Chen Y, Liu Q, Guo D. Emerging coronaviruses: Genome structure, replication, and pathogenesis. J Med Virol. 2020;92(4):418-23.

5. Benvenuto D, Giovanetti M, Ciccozzi A, Spoto S, Angeletti S, Ciccozzi M. The 2019-new coronavirus epidemic: Evidence for virus evolution. J Med Virol. 2020;92(4):455-59.

6. Zhang MQ, Wang $X H$, Chen $Y L$, Zhao, KL, Cai, $Y Q$, An, CL, et al. Clinical features of coronavirus disease 2019pneumonia in the early stage from a fever clinic in Beijing. Zhonghua Jie He He Hu Xi Za Zhi. 2020;43(3):215-8.

7. Huang C, Wang Y, Li X, Ren L, Zhao J, Hu Y, et al. Clinical features of patients infected with 2019 novel coronavirus in Wuhan, China.Lancet.2020;395(10223):497-506.

8. Chen N, Zhou M, Dong X, Qu J, Gong F, Han Y, et al. Epidemiological and clinical characteristics of 99 cases of 2019 novel coronavirus pneumonia in Wuhan, China: a descriptive study. Lancet. 2020;395(10223):507-13. 
9. Huang R, Xia J, Chen Y, Shan C, Wu C. A family cluster of SARS-CoV-2 infection involving 11 patients in Nanjing, China. Lancet Infect Dis. 2020;20(5):534-35.

10. Wang J, Zhu X, Xu Z, Yang G, Mao G, Jia Y, et al. Clinical and CT findings of COVID-19: differences among three age groups. BMC Infect Dis. 2020; 20(1):434.

11. Leung JM, Yang CX, Tam A, Shaipanich T, Hackett TL, Singhera GK, et al. ACE-2 expression in the small airway epithelia of smokers and COPD patients: implications for COVID-19. Eur Respir J. 2020. https://doi.org/10. 1183/13993003.00688-2020.

12. Li YX, Wu W, Yang T, Fu YM, Feng QM, Ye JM. Characteristics of peripheral blood leukocyte differential counts in patients with COVID-19. Zhonghua Nei Ke Za Zhi.2020;59(5):372-4.

13. Leist SR, Jensen KL, Baric RS, Sheahan TP. Increasing the translation of mouse models of MERS coronavirus pathogenesis through kinetic hematological analysis. PLoS One.2019; 14(7): e0220126.

14. Tabata S, Imai K, Kawano S, Ikeda M, Kodama T, Miyoshi K, et al. Clinical characteristics of COVID-19 in 104 people with SARS-CoV-2 infection on the Diamond Princess cruise ship: a retrospective analysis. Lancet Infect Dis.2020. https://doi.10.1016/S1473-3099(20)30482-5.

15. Liu J, Li S, Liu J, Liang B, Wang X, Wang H, et al. Longitudinal characteristics of lymphocyte responses and cytokine profiles in the peripheral blood of SARS-CoV-2 infected patients. EBioMedicine. 2020;55:102763.

16. Leist SR, Jensen KL, Baric, RS, Sheahan, TP, et al. Increasing the translation of mouse models of MERS coronavirus pathogenesis through kinetic hematological analysis. PLoS One.2019;14(7):e0220126.

17. Wang D, Hu B, Hu C, Zhu F, Liu X, Zhang, J, et al. Clinical Characteristics of 138 Hospitalized Patients With 2019 Novel Coronavirus-Infected Pneumonia in Wuhan, China.JAMA.2020. https://doi.10.1001/jama.2020.1585.

18. Chen N, Zhou M, Dong X, Qu J, Gong F, Han Y, et al. Epidemiological and clinical characteristics of 99 cases of 2019 novel coronavirus pneumonia in Wuhan, China: a descriptive study. Lancet. 2020;395(10223):507-513.

19. Bermejo-Martin JF, Almansa R, Menéndez R, Mendez R, Kelvin DJ, Torres A. Lymphopenic community acquired pneumonia as signature of severe COVID-19 infection. J Infect. 2020;80(5):23-4.

20. Torres A, Ceccato A, Ferrer M, Gabarrus A, Sibila O, Cilloniz C, et al. Effect of Corticosteroids on CReactive Protein in Patients with Severe Community-Acquired Pneumonia and High Inflammatory Response: The Effect of Lymphopenia. J Clin Med. 2019;8(9):1461.

21. Pan $Y$, Guan $H$, Zhou S, Wang, Y, Li, Q, Zhu, T, et al. Initial CT findings and temporal changes in patients with the novel coronavirus pneumonia (2019-nCoV): a study of 63 patients in Wuhan, China. Eur Radiol. 2020;30(6):3306-9.

22. Zhou S, Wang Y, Zhu T, Xia L. CT Features of Coronavirus Disease 2019 (COVID-19) Pneumonia in 62 Patients in Wuhan, China. AJR Am J Roentgenol. 2020;214(6):1287-94.

23. Wu J, Wu X, Zeng W, Guo D, Fang Z, Chen L, et al. Chest CT Findings in Patients With Coronavirus Disease 2019 and Its Relationship With Clinical Features. Invest Radiol. 2020;55(5):257-61. 
24. Wan Y, Shang J, Graham R, Baric RS, Li F. Receptor Recognition by the Novel Coronavirus from Wuhan: an Analysis Based on Decade-Long Structural Studies of SARS Coronavirus. J Virol. 2020;94(7): e00127-20.

\section{Figures}

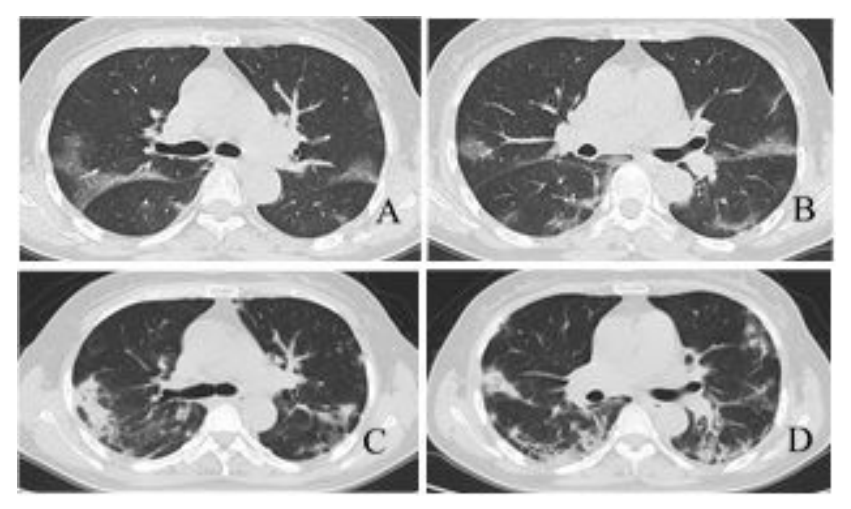

Figure 1

Chest CT images (January 30, 2020) (A, B) Chest CT images from a 50-60 year-old woman showing multiple bilateral ground-glass opacity in the lung lobes, which are distributed along the bronchial blood vessel bundles. In the lesions, vascular shadows are thickened and grid-like changes are accompanied by signs of bronchial inflation. Cord shadows and pleural thickening are seen below the pleura. (C, D) Multiple fiber strand shadow formation after 14 days of treatment.

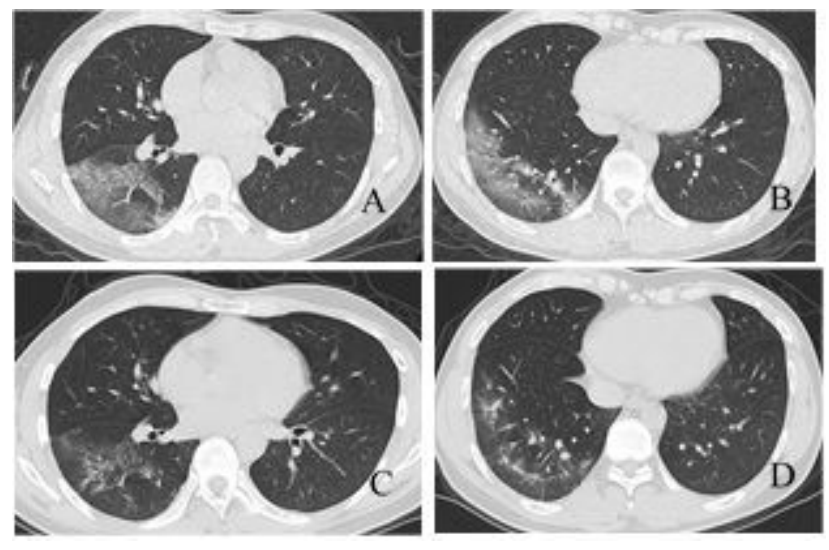

Figure 2

Chest CT images from a 30-40 year-old man, (A₫B) $₫$ Ground-glass opacity in the basal segment of the right lower lobe, peripheral subpleural distribution, thickening of blood vessels and rigid distribution in the

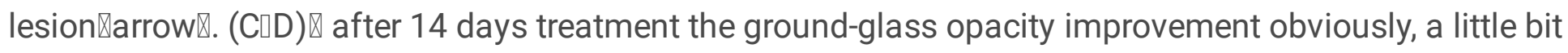
of fiber shadow hand down in the right lower lung, the Lesion parallel to the pleura,formation of the socalled "down pleural line" (black arrow). 